\section{LAS ESTERILIZACIONES FORZADAS, LOS DERECHOS REPRODUCTIVOS Y EL CONSENTIMIENTO INFORMADO}

\author{
FORCED STERILIZATIONS, REPRODUCTIVE \\ RIGHTS AND INFORMED CONSENT
}

\author{
Germán Málaga ${ }^{1,2, a}$
}

Sr. Editor. En el artículo de Gianella se hizo una revisión de la ideología del consentimiento médico informado, las bases de su desarrollo e implementación sustentadas en un principio básico de la medicina, cómo es la autonomía, y cómo este representa la aplicación de los derechos humanos en salud ${ }^{(1)}$.

Entre los años 1993 a 1999 se realizaron en el Perú, 314605 (2) esterilizaciones quirúrgicas, las cuales obedecieron a la implementación de una política de estado de "lucha contra la pobreza", en la que se establecieron metas que privilegiaban las opciones quirúrgicas sobre los métodos reversibles. Para el logro de objetivos, se ejerció presión psicológica sobre la población, manipulación, amedrentamiento, condicionamiento a cambio de incentivos como víveres o inclusión en los programas de salud nacionales a los que tenían pleno derecho. Asimismo, se establecieron incentivos para el personal de salud "más productivo" $y$, en algunos casos, estos fueron intimidados con el cese de sus contratos en caso no sumaran esfuerzos para alcanzar las metas propuestas.

Según la Defensoría del Pueblo (3) y el Movimiento Amplio de Mujeres ${ }^{(2)}$, más del $65 \%$ de las mujeres esterilizadas eran analfabetas o tenían primaria incompleta, y gran parte de ellas eran quechua hablantes, es decir, difícilmente pudieron entender los consentimientos informados. A ello se agrega que se implementaron "campañas de esterilización" sin sesiones informativas. Con lo cual se puede afirmar que estas campañas se hicieron sin que ocurriera un genuino consentimiento, lo que se refleja en estudios que señalan que hasta el $90 \%$ de las pacientes intervenidas tomaron decisiones bajo presión (2).

\footnotetext{
1 Unidad de Conocimiento y Evidencia, Facultad de Medicina, Universidad Peruano Cayetano Heredia.

2 CRONICAS Centro de Excelencia en Enfermedades Crónicas, Universidad Peruana Cayetano Heredia. Lima, Perú.

a Médico internista, magíster en Medicina

Recibido: 07-07-13 Aprobado: 17-07-13
}

Citar como: Málaga G. Las esterilizaciones forzadas, los derechos reproductivos y el consentimiento informado [carta]. Rev Peru Med Exp Salud Publica. 2013;30(3):521-2.
Si a lo referido, agregamos que las intervenciones fueron realizadas en establecimientos con infraestructura inapropiada, en condiciones de escasa higiene, sin evaluación preoperatoria, y hasta en el $50 \%$ de los casos, sin control posoperatorio, ni soporte posterior que permitiera resolver dudas y las consecuentes afecciones orgánicas y psicológicas suscitadas ${ }^{(4)}$, tenemos que la situación fue complicada no solo desde la perspectiva de derechos fundamentales, sino que las condiciones básicas de atención medica fueron también vulneradas.

Estos hechos fueron dados a conocer esencialmente por organismos defensores de los derechos humanos y por escasos medios de comunicación, y pese a que fue denunciado, estudiado y documentado por instituciones como la Defensoría del Pueblo, por organizaciones no gubernamentales defensoras de los derechos humanos y por la Conferencia Episcopal, fue solo después del año 2000 que se interesaron por el tema instituciones como el Colegio Médico del Perú y el Congreso de la Republica ${ }^{(5)}$.

A pesar de ello, nunca hubo una asunción de responsabilidades. La discusión y alusiones al tema, siempre se hicieron desde la perspectiva política, pero no hubo un reconocimiento de participación equivocada ni menos una intención de tomar las medidas para evitar que situaciones tan flagrantemente violatorias de derechos vuelvan a ocurrir. El hecho de que se propale en su revista artículos como el referido (1) o se hable de la toma de decisiones medicas compartidas como forma efectiva de defender la autonomía ${ }^{(6)}$, claramente abren esperanzas de que en el Perú practiquemos una medicina en la que el paciente siendo el centro del acto médico reciba la atención con pleno respeto de sus derechos.

Fuentes de financiamiento: GM está afiliado a CRONICAS Centro de Excelencia en Enfermedades Crónicas de la Universidad Peruana Cayetano Heredia, el cual es financiado por el National Heart, Lung and Blood Institute (NHLBI), National Institute of Health $(\mathrm{NIH})$, Department of Health and Human Services.

Conflictos de interés: el autor declara no tener conflictos de interés.

\section{REFERENCIAS BIBLIOGRÁFICAS}

1. Gianella G. Los derechos humanos y el consentimiento informado en la práctica clínica: más allá del derecho a la salud. Rev Peru Med Exp Salud Publica. 2013;30(2):315-9.

2. Succar Rahme J, García Trovato M, Reyes Solari E, Supa Huamán H. Comisión Especial sobre actividades de Anticoncepción Quirúrgica Voluntaria (AQV). Informe Final [Internet]. Lima: Movimiento Amplio de Mujeres Línea Fundacional; 2002 [citado el 14 de mayo del 2013]. Disponible en: http:// www.mamfundacional.org/ef/Informe-Final.pdf

3. La Anticoncepción quirúrgica voluntaria. Casos investigados por la defensoría del pueblo. 1998. http://www.corteidh. or.cr/tablas/10636a.pdf 
4. Ballón A. El caso peruano de esterilización forzada. Ensayo fotográfico de Alejandra Ballón G. distrito de Huancabamba, Piura, Perú (2012) [Internet]. Lima: Archivo PNSRPF; c2011-2013 [citado el 14 de mayo del 2013]. Disponible en: http://1996pnsrpf2000.files.wordpress.com/2011/07/ensayofotografico-ef-hbba.pdf

5. Subcomisión investigadora de personas e instituciones involucradas en las acciones de anticoncepción quirúrgica voluntaria $(\mathrm{AQV})$. Informe final sobre la aplicación de la anticoncepción quirúrgica voluntaria (AQV) en los años 1990-2000. Lima: Congreso de la República del Perú; 2002. Disponible en: http://www.trdd.org/PERU_Informe_Final_AQV.pdf.

6. Zevallos-Palacios C, Quispe R, Mongilardi N, Montori VM, Málaga G. La participación del paciente en la toma de decisiones clínicas como estrategia para salvaguardar sus derechos [carta]. Rev Peru Med Exp Salud Publica. 2013;30(2):363-4.

Correspondencia: Germán Málaga

Dirección: Calle Chavin 159, CC Monterrico, Lima 33, Perú. Teléfono: (51) 992768300

Correo electrónico: german.malaga@upch.pe

\section{APLICACIÓN DE UN SISTEMA DE INFORMACIÓN ELECTRÓNICO AL PROGRAMA DE TAMIZAJE DE CÁNCER DE CUELLO UTERINO}

\author{
APPLICATION OF AN ELECTRONIC \\ INFORMATION SYSTEM TO THE CERVICAL \\ CANCER SCREENING PROGRAM
}

\begin{abstract}
Javier Vargas-Herrera ${ }^{1,2, a}$, Javier Manrique ${ }^{3, b}$, María del Carmen Nuñez ${ }^{3, c}$, Paula Sánchez ${ }^{4, d}$, ${\text { María Miraval }{ }^{1, e}, \text { Mercy Merejildo }{ }^{1, f}, \text { Violeta Puntas }}^{5, c}$
\end{abstract}

Señor Editor. Los programas de tamizaje de cáncer de cuello uterino (CACU) han logrado un impacto significativo en la disminución de la incidencia y la mortalidad por este cáncer en los países desarrollados,

\footnotetext{
Centro Nacional de Salud Pública, Instituto Nacional de Salud. Lima, Perú. Facultad de Medicina, Universidad Nacional Mayor de San Marcos. Lima Perú. Instituto Nacional de Enfermedades Neoplásicas. Lima, Perú.

Dirección de Salud II. Lima, Perú.

Red de Salud San Juan Villa María, Dirección de Salud II. Lima, Perú.

Médico epidemiólogo; ${ }^{\mathrm{b}}$ médico oncólogo; ${ }^{\mathrm{c}}$ licenciada en obstetricia; ${ }^{\mathrm{d}}$ citotecnóloga; ${ }^{\mathrm{c}}$ médico patólogo; ${ }^{\mathrm{f}}$ tecnólogo médico

Recibido: 10-07-13 Aprobado: 17-07-13
}

Citar como: Vargas-Herrera J, Manrique J, Nuñez MC, Sánchez P, Miraval M, Merejildo M, et al. Aplicación de un sistema de información electrónico al programa de tamizaje de cáncer de cuello uterino [carta]. Rev Peru Med Exp Salud Publica. 2013;30(3):522-4. no así en los países de bajos y medianos ingresos (1). Los factores más importantes que se han relacionado con la baja efectividad de los programas de tamizaje en los países en vías de desarrollo son: la deficiente organización, la baja cobertura; la baja calidad de las muestras obtenidas; los errores en el diagnóstico citológico, y el escaso seguimiento de los casos encontrados. Incluso, este último se ha asociado con un incremento moderado de la mortalidad ${ }^{(2,3)}$. A pesar de estas dificultades, la Organización Mundial de la Salud (OMS) considera que los programas de tamizaje siguen siendo la mejor alternativa para la prevención y el control del CACU, frente al uso de las vacunas contra el virus del papiloma humano (VPH) que abarca la prevención contra los tipos 16 y 18 que se relacionan solo con el $70 \%$ de los cánceres de cuello uterino.

Actualmente, el sistema de información del programa de tamizaje de $\mathrm{CACU}$, que funciona en los establecimientos de salud de primer nivel de atención del Perú, está basado en formatos de registro manual, lo cual dificulta el procesamiento y análisis de la información, al tiempo que limita el seguimiento de los casos. Usualmente, los obstetras o las obstetrices obtienen las muestras de citología cervicouterina, registran los datos de las pacientes en un libro de registro y envían las muestras a un laboratorio de referencia de citología local, cada siete días. Estos laboratorios entregan los reportes de resultados en un tiempo que varía de 7 a 30 días, pero que puede llegar a ser, en algunos casos, mayor a 100 días. Este tipo de sistema condiciona problemas de identificación de las muestras o de las pacientes, pérdidas de las muestras 0 de los reportes de resultados, y retraso en la comunicación de resultados a las pacientes.

En el Perú, el uso de un sistema de información electrónico en línea denominado NETLAB ha permitido reducir sustancialmente el tiempo de entrega de reportes de resultados de laboratorio. Este sistema permite, además, monitorizar el estado en que se encuentra la muestra y elimina la posibilidad de que alguna se quede sin resultado o sin una explicación acerca del motivo por el cual no se procesó ${ }^{(4)}$. Su empleo en los programas de tamizaje de CACU podría ayudar a ordenar la información en una base de datos, facilitar el seguimiento de los casos detectados, contar con un historial de los resultados de las pruebas de las pacientes, reducir el tiempo de entrega de resultados y proporcionar una plataforma sobre la que se pueden incluir nuevas pruebas como la inspección visual con ácido acético o la detección del VPH, en un marco de confidencialidad. 